\title{
Classical-Field Method for Time Dependent Bose-Einstein Condensed Gases
}

\author{
Alice Sinatra, Carlos Lobo,* and Yvan Castin \\ Laboratoire Kastler Brossel, 24 Rue Lhomond, 75231 Paris Cedex 05, France
}

(Received 8 January 2001; published 2 November 2001)

\begin{abstract}
We propose a method to study the time evolution of Bose-Einstein condensed gases perturbed from an initial thermal equilibrium, based on the Wigner representation of the $N$-body density operator. We show how to generate a collection of random classical fields sampling the initial Wigner distribution in the number conserving Bogoliubov approximation. The fields are then evolved with the time dependent Gross-Pitaevskii equation. We illustrate the method with the damping of a collective excitation of a one-dimensional Bose gas.
\end{abstract}

DOI: $10.1103 /$ PhysRevLett.87.210404

Since the first experimental demonstrations of BoseEinstein condensation in atomic gases [1], the role played by finite temperature effects in the physics of BoseEinstein condensed alkali gases has drawn increasing attention. For example, in thermal equilibrium, both the spatial density of the condensate atoms [2] and the distribution of number of particles in the condensate [3] are modified due to the presence of the thermal atoms. Similarly, we must take into account the noncondensed atoms in order to explain time dependent phenomena such as the damping and frequency shifts of collective modes $[4,5]$ or the evolution of the recently created vortices [6], where the dissipation of the condensate motion is provided by the noncondensed atoms [7].

The widely used Gross-Pitaevskii equation, the nonlinear Schrödinger equation for the condensate wave function, is not able to describe these effects since it neglects the interaction between the condensate and the noncondensed atoms [8]. One possibility to go beyond the GrossPitaevskii equation is to use Bogoliubov theory, which is a perturbative method valid for a small noncondensed fraction. The Bogoliubov method can be applied to thermal equilibrium but also to time dependent situations: in a $\mathrm{U}(1)$ symmetry breaking point of view, to zeroth order one solves the time dependent Gross-Pitaevskii equation for the condensate field $\psi_{0}(\vec{r}, t)$, to first order one linearizes the quantum field equations around the classical field $\psi_{0}(\vec{r}, t)$ to get the dynamics of noncondensed particles, and to second order one includes the backaction of noncondensed particles on the condensate. However, in this way one can predict only small corrections to the Gross-Pitaevskii equation. Additionally, if the number of noncondensed particles increases during the evolution of the system, the Bogoliubov approach is valid only for short times [9]. Another existing approach is the mean field HartreeFock-Bogoliubov approximation. This approach is known however to present consistency problems and is still the object of research [10].

In this Letter we propose an alternative method to study the time evolution of Bose condensed gases perturbed from an initial thermal equilibrium, based on the classical field approximation in the Wigner representation, the so-called
PACS numbers: 03.75.Fi, 05.10.Gg, 42.50.-p

truncated Wigner approximation. The classical field approximation amounts to evolving a set of initially randomly distributed atomic fields with the usual Gross-Pitaevskii equation, with the crucial point that the field is now the whole matter field, not simply the condensate field. This approximation has been used already in the Glauber- $P$ representation to study the formation of the condensate and it is expected to be correct for modes having a large occupation number $[11,12]$. For such modes this approximation is known, however, to be most accurate in the Wigner representation as quantum noise is mimicked by classical noise in the initial state [13]. In addition in such a representation, the approximation is valid also for modes with a small occupation number as long as their evolution is well described by a quadratic approximation to the Hamiltonian, as in the Bogoliubov approach. The classical field approximation is indeed exact in the Wigner representation for a quadratic Hamiltonian. The truncated Wigner approximation is however expected to fail in describing the relaxation of the gas to the correct thermal equilibrium, and one has to supplement the Gross-Pitaevskii equation by kinetic equations for the high energy modes [11].

The main result of this Letter is the construction of an algorithm to approximately sample the Wigner distribution at thermal equilibrium, using the number conserving Bogoliubov theory [14-16]. This was the missing block for the implementation of the truncated Wigner approach to study the dynamics of low temperature Bose condensed gases. We apply our method to the damping of the breathing mode of a 1D condensate in a harmonic trap, which exemplifies the superiority of the truncated Wigner over the time dependent Bogoliubov approach.

We recall briefly the number conserving Bogoliubov approach that we use to describe the initial thermal equilibrium state of the gas. The total number of particles of the gas is supposed to be fixed and equal to $N$. The existence of the macroscopically populated mode $\phi(\vec{r})$ motivates the splitting of the atomic field operator:

$$
\hat{\psi}(\vec{r})=\phi(\vec{r}) \hat{a}_{\phi}+\hat{\psi}_{\perp}(\vec{r}),
$$

where $\phi$ is the condensate wave function and $\hat{a}_{\phi}$ annihilates a particle in the mode $\phi$. The general idea is to 
eliminate the annihilation operator for the condensate. Unlike the usual approach, which replaces $\hat{a}_{\phi}$ by a fixed complex number, we keep the operator nature of $\hat{a}_{\phi}$ and write it in terms of the condensate phase operator $\hat{A}_{\phi}$ and the number of condensate particles operator $\hat{N}_{0}$ as

$$
\hat{a}_{\phi}=\hat{A}_{\phi} \hat{N}_{0}^{1 / 2} \text {. }
$$

The modulus of $\hat{a}_{\phi}$ can be reexpressed as a function of the number of noncondensed particles:

$$
\hat{N}_{0}=\hat{N}-\int d \vec{r} \hat{\psi}_{\perp}^{\dagger}(\vec{r}) \hat{\psi}_{\perp}(\vec{r}) .
$$

Since $\hat{A}_{\phi}$ and $\hat{A}_{\phi}^{\dagger}$ are commuting unitary operators [17]

$$
\hat{A}_{\phi} \hat{A}_{\phi}^{\dagger}=\hat{A}_{\phi}^{\dagger} \hat{A}_{\phi}=\mathrm{Id}
$$

commuting also with $\hat{\psi}_{\perp}, \hat{\psi}_{\perp}^{\dagger}$, we realize that the Hamiltonian can be expressed as a function of the fields $\hat{\Lambda}(\vec{r})=$ $\hat{A}_{\phi}^{\dagger} \hat{\psi}_{\perp}(\vec{r})$ and $\hat{\Lambda}^{\dagger}(\vec{r})$. In the Bogoliubov approximation we can then quadratize the Hamiltonian in terms of $\hat{\Lambda}$ : the terms linear in $\hat{\Lambda}$ vanish when $\phi$ solves the time independent Gross-Pitaevskii equation:

$H_{\mathrm{gp}} \phi \equiv\left[-\frac{\hbar^{2}}{2 m} \Delta+U(\vec{r})+N g|\phi|^{2}-\mu\right] \phi=0$,

where $m$ is the atomic mass, $U(\vec{r})$ is the trapping potential, $g$ is the atomic coupling constant, and $\mu$ is the chemical potential. The resulting quadratic form is the Bogoliubov Hamiltonian

$$
\hat{H}_{\text {quad }}(\hat{\Lambda})=\int d^{3} \vec{r} \frac{1}{2}\left(\hat{\Lambda}^{\dagger},-\hat{\Lambda}\right) \cdot \mathcal{L}\left(\begin{array}{c}
\hat{\Lambda} \\
\hat{\Lambda}^{\dagger}
\end{array}\right),
$$

where the differential operator $\mathcal{L}$ is given by

$$
\mathcal{L}=\left(\begin{array}{cc}
H_{\mathrm{gp}}+Q N g|\phi|^{2} Q & Q N g \phi^{2} Q^{*} \\
-Q^{*} N g\left(\phi^{*}\right)^{2} Q & -H_{\mathrm{gp}}^{*}-Q^{*} N g|\phi|^{2} Q^{*}
\end{array}\right) .
$$

Here $Q \equiv \mathrm{Id}-|\phi\rangle\langle\phi|$ projects orthogonally to $\phi$.

The initial $N$-body density operator of the gas at temperature $T$ is then approximated by

$$
\hat{\sigma}(t=0) \simeq \frac{1}{Z} \exp \left[-\frac{1}{k_{B} T} \hat{H}_{\text {quad }}(\hat{\Lambda})\right] .
$$

The Wigner distribution associated to the $N$-body density operator $\hat{\sigma}$ is a functional of a complex classical field $\psi(\vec{r})$. It is defined as the Fourier transform of a characteristic function $\chi$ :

$$
\begin{gathered}
W(\psi) \equiv \int \mathcal{D}^{2} \gamma \chi(\gamma) e^{\int \gamma^{*} \psi-\gamma \psi^{*}} \\
\chi(\gamma)=\operatorname{Tr}\left[\hat{\sigma} e^{\int \gamma \hat{\psi}^{\dagger}-\gamma^{*} \hat{\psi}}\right],
\end{gathered}
$$

where $\int \mathcal{D}^{2} \gamma$ stands for the functional integral over the real and the imaginary part of the complex classical field $\gamma(\vec{r})$ and the integrals in the exponent are over space.

We now proceed with the analytical calculation of the Wigner distribution of the density operator (8). As we did with the atomic field operator, we split all the classical fields into components parallel and orthogonal to the condensate mode $\phi$, e.g., $\psi(\vec{r})=\psi_{\|} \phi(\vec{r})+\psi_{\perp}(\vec{r})$.

It will prove convenient to use the commutation of $\hat{a}_{\phi}$ with $\hat{\psi}_{\perp}$ to rewrite the characteristic function as

$$
\chi(\gamma)=\frac{1}{2}\left\langle\left\{e^{\gamma_{\|} \hat{N}_{0}^{1 / 2} \hat{A}_{\phi}^{\dagger}-\text { H.c. }}, e^{\int \gamma_{\perp} \hat{\Lambda}^{\dagger} \hat{A}_{\phi}^{\dagger}-\text { H.c. }}\right\}\right\rangle,
$$

where $\{$,$\} stands for the anticommutator and the brackets$ denote the expectation value in the density operator $\hat{\sigma}$. We then introduce an approximation for $\hat{N}_{0}$ in (11) replacing the operator $\hat{N}$ in Eq. (3) by its actual value $N$ in the gas:

$$
\hat{N}_{0} \simeq N-\int \hat{\Lambda}^{\dagger} \hat{\Lambda} \text {. }
$$

As a consequence the phase operators $\hat{A}_{\phi}, \hat{A}_{\phi}^{\dagger}$ commute with all the operators appearing in $\chi$. We now calculate the trace in (11) using a simultaneous eigenbasis of the phase operator $\hat{A}_{\phi}$, with eigenvalue $e^{i \theta}$, and of the Bogoliubov Hamiltonian whose eigenvalues depend on the occupation numbers of elementary excitations but not on the phase $\theta$. The form obtained for $W$ clearly preserves the $\mathrm{U}(1)$ symmetry:

$$
W(\psi) \simeq \int_{0}^{2 \pi} \frac{d \theta}{2 \pi} W_{0}\left(\psi e^{i \theta}\right) .
$$

$W_{0}$ is the $\theta=0$ component of the Wigner distribution. By integrating over the real and the imaginary parts of $\gamma_{\|}$and by using the cyclic properties of the trace we get

$W_{0}(\psi)=\delta\left(\psi_{\|}^{I}\right) \operatorname{Wig}_{\Lambda}\left[\frac{1}{2}\left\{\delta\left(\psi_{\|}^{R}-\hat{N}_{0}^{1 / 2}\right), \hat{\sigma}\right\}\right]\left(\psi_{\perp}\right)$, where $\psi_{\|}^{R, I}$ are the real and imaginary parts of $\psi_{\|}$. The expression $\operatorname{Wig}_{\Lambda}[f(\hat{\Lambda})]$ denotes the Wigner transform with respect to $\hat{\Lambda}$ of any function $f$ of $\hat{\Lambda}$. It is obtained from (9) and (10) by the substitutions:

$$
\hat{\sigma} \rightarrow f(\hat{\Lambda}) \quad \hat{\psi} \rightarrow \hat{\Lambda} \quad \psi \rightarrow \psi_{\perp} \quad \gamma \rightarrow \gamma_{\perp} .
$$

It is convenient to rewrite $W_{0}(\psi)$ as a quasiprobability distribution for $N_{0} \equiv \psi_{\|}^{*} \psi_{\|}$and $\psi_{\perp}$ :

$$
P\left(N_{0}, \psi_{\perp}\right)=\operatorname{Wig}_{\Lambda}\left[\frac{1}{2}\left\{\delta\left(N_{0}-\hat{N}_{0}\right), \hat{\sigma}\right\}\right]\left(\psi_{\perp}\right) .
$$

The first insight into (16) can be obtained by looking at its marginals. By integrating over $N_{0}$,

$$
P\left(\psi_{\perp}\right) \equiv \int d N_{0} P\left(N_{0}, \psi_{\perp}\right)=\operatorname{Wig}_{\Lambda}(\hat{\sigma})\left(\psi_{\perp}\right),
$$

we obtain the Wigner distribution for noncondensed modes, which is a Gaussian [18]:

$$
\begin{gathered}
P\left(\psi_{\perp}\right) \propto \exp \left\{-\int d^{3} \vec{r}\left(\psi_{\perp}^{*}, \psi_{\perp}\right) \cdot M\left(\begin{array}{c}
\psi_{\perp} \\
\psi_{\perp}^{*}
\end{array}\right)\right\}, \\
M \equiv\left(\begin{array}{cc}
1 & 0 \\
0 & -1
\end{array}\right) \tanh \frac{\mathcal{L}}{2 k_{B} T}
\end{gathered}
$$

where $\mathcal{L}$ is the Bogoliubov operator (7). The other marginal is obtained by integrating (16) over $\psi_{\perp}$ :

$$
P\left(N_{0}\right) \equiv \int \mathcal{D}^{2} \psi_{\perp} P\left(N_{0}, \psi_{\perp}\right)=\operatorname{Tr}\left[\delta\left(N_{0}-\hat{N}_{0}\right) \hat{\sigma}\right] .
$$


$P\left(N_{0}\right)$ is then simply the probability distribution of the number of particles in the condensate [19].

Let us now discuss the limits of validity of (16). The spectrum of $\hat{N}_{0}$ contains integer numbers only so that $\delta\left(N_{0}-\hat{N}_{0}\right)$ in Eq. (16) vanishes when $N_{0}$ is not integer. As a function of $N_{0}, P\left(N_{0}, \psi_{\perp}\right)$ is therefore a "comb" of delta functions centered on integer values. The exact Wigner distribution should be instead a smooth function of its variables. The comb artifact comes from the approximation (12) that we have made on the condensate mode. Our description is sensible if there are many peaks within the width of the distribution of $N_{0}$ for a given $\psi_{\perp}$. The variance of $N_{0}$ for a given $\psi_{\perp}$, that is, the conditional variance of $N_{0}$, should then satisfy

$$
\operatorname{Var}\left(N_{0} \mid \psi_{\perp}\right) \gg 1 .
$$

We estimate this variance for a "typical" $\psi_{\perp}$ by taking the mean of $\operatorname{Var}\left(N_{0} \mid \psi_{\perp}\right)$ over the probability distribution of $\psi_{\perp}$ given in (18):

$$
\left\langle\operatorname{Var}\left(N_{0} \mid \psi_{\perp}\right)\right\rangle_{\psi_{\perp}}=\frac{1}{8} \operatorname{Tr}_{\perp}\left[\mathrm{Id}-M^{2}\right],
$$

where the trace is over the Bogoliubov modes. For the modes with energy much larger than $k_{B} T$ one has $M^{2} \simeq$ Id, while for the modes with energy lower or equal to $k_{B} T$ one has $M^{2} \ll \mathrm{Id}$, so that

$$
\left\langle\operatorname{Var}\left(N_{0} \mid \psi_{\perp}\right)\right\rangle_{\psi_{\perp}} \simeq \frac{1}{4} \mathcal{N}_{\mathrm{th}}
$$

where $\mathcal{N}_{\text {th }}$ is the number of thermally populated modes which we require to be large. In practice $k_{B} T$ should be high enough: for a condensate in an isotropic harmonic trap of frequency $\omega$, we should have $k_{B} T \gg \hbar \omega$.

The next point is to sample the Wigner function (16). We proceed in two steps: (i) First we sample $\psi_{\perp}$ irrespectively to the value $N_{0}$ according to the Gaussian distribution (18) with a stochastic field method $[18,20]$. (ii) We have to sample the conditional distribution of $N_{0}$ for a fixed $\psi_{\perp}$ :

$$
P\left(N_{0} \mid \psi_{\perp}\right)=P\left(N_{0}, \psi_{\perp}\right) / P\left(\psi_{\perp}\right) .
$$

It turns out that the width in $N_{0}$ of the distribution $P\left(N_{0} \mid \psi_{\perp}\right)$ is much narrower than the width of $P\left(N_{0}\right)$ when the condition (21) is satisfied. In fact, as soon as a sufficient number of modes is populated, the following inequality holds involving the number of modes $\mathcal{N}_{\text {th }}$ with energy lower or equal to $k_{B} T$, the average of the number of particles out of the condensate $\left\langle N-\hat{N}_{0}\right\rangle$, and its variance $\operatorname{Var}\left(N-\hat{N}_{0}\right)$ :

$$
\mathcal{N}_{\text {th }} \leq\left\langle N-\hat{N}_{0}\right\rangle \ll \operatorname{Var}\left(N-\hat{N}_{0}\right)=\operatorname{Var}\left(N_{0}\right) .
$$

We then replace (24) with a delta distribution centered on the mean value of $N_{0}$ for a fixed $\psi_{\perp}$ :

$\operatorname{Mean}\left(N_{0} \mid \psi_{\perp}\right)=C-\frac{1}{2} \int\left(\psi_{\perp}^{*}, \psi_{\perp}\right)\left[\operatorname{Id}-M^{2}\right]\left(\begin{array}{l}\psi_{\perp} \\ \psi_{\perp}^{*}\end{array}\right)$,

where $C$ is a constant [21].

Finally, for each randomly generated $\psi_{\perp}$ and $N_{0}$ we are now able to form the total field $\psi$ as

$$
\psi=N_{0}^{1 / 2}\left(\phi+\frac{\phi^{(2)}}{N}\right)+\psi_{\perp} .
$$

In (26) the function $\phi^{(2)} / N$ is a correction to the condensate wave function induced by the backaction of noncondensed particles onto the condensate. This correction has to be included since it gives a contribution to observables on the same order as $\psi_{\perp}$ [16]. We calculate $\phi^{(2)} / N$ using the procedure of [18].

In the classical field approximation each field $\psi$ evolves according to the time dependent Gross-Pitaevskii equation $[13,18]$ :

$$
i \hbar \partial_{t} \psi=\left[-\frac{\hbar^{2}}{2 m} \Delta+U(\vec{r}, t)+g|\psi|^{2}\right] \psi
$$

The average of an observable at time $t$ will be obtained by averaging over many stochastic classical fields $\psi(t)$.

We now give an illustration of our method. In Fig. 1 we show density oscillations in the center of a 1D Bose condensed gas induced by an abrupt change of the harmonic trap frequency $\omega \rightarrow 0.8 \omega$. While the Gross-Pitaevskii equation for the condensate field predicts undamped oscillations, we get damping at finite temperature when we average over stochastic realizations in the Wigner method [22]. The time dependent Bogoliubov theory in [16] correctly predicts the damping at short times but gives unphysically large oscillations at longer times. We understand this failure as due to the growth in time of the number of noncondensed particles and of the condensate wave-function correction $\phi^{(2)}$. This growth invalidates the linearized treatment at the basis of the time dependent Bogoliubov approach [9], at a relatively short time here because of the small number of particles. On the contrary, in the truncated Wigner approach, no linearized treatment is performed and the whole field $\psi$ is evolved according to the fully nonlinear equation (27).

In Fig. 1 a spatial grid of 64 points has been used for discretization in the numerical implementation of our algorithm. We have tested that the results are unchanged by doubling the number of points in the grid [23].
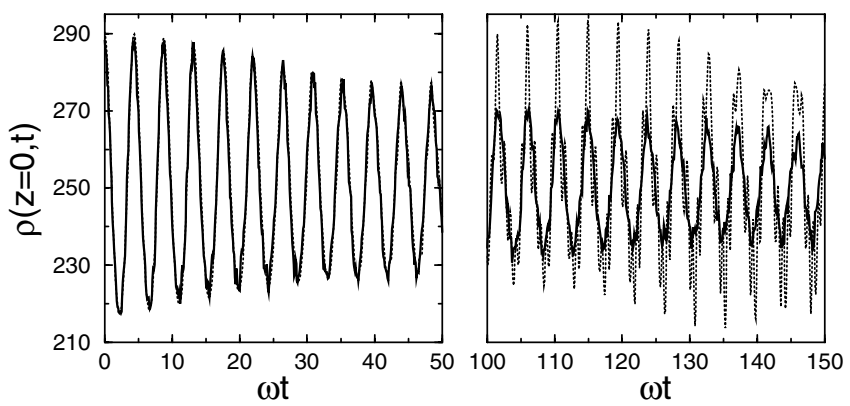

FIG. 1. Damped oscillations of the mean density in the center of a 1D condensed cloud in a harmonic trap after an abrupt change of the trap frequency $\omega \rightarrow 0.8 \omega . k_{B} T=30 \hbar \omega, \mu=$ $3.1 \hbar \omega$, and $N=10^{3}$. Solid line: Wigner simulation. Dotted curve: Bogoliubov theory. $\rho$ is in units of $(m \omega / \hbar)^{1 / 2}$. 
In conclusion, we have developed an efficient algorithm for approximate stochastic sampling of the Wigner representation of the $\mathrm{N}$-body density matrix of a Bose condensed gas in thermal equilibrium. The initial distribution of atomic fields $\psi$ given by this sampling can then be evolved in the classical field (truncated Wigner) approximation to study the response of the gas to an excitation. As far as time independent situations are concerned, our approach is equivalent to the number conserving Bogoliubov approach. In the time dependent case, however, unlike the Bogoliubov approach, the classical field evolution is fully nonlinear both for the condensate and the noncondensed particles, which extends the applicability of the method to longer evolution times.

We acknowledge useful discussions with Anthony Leggett, Yuri Kagan, Gora Shlyapnikov, and Crispin Gardiner. LKB is a unité de recherche de l'ENS et de l'Université Paris 6, associée au CNRS. This work was partially supported by National Computational Science Alliance under DMR $990016 \mathrm{~N}$.

*Present address: University of Illinois at UrbanaChampaign, 1110 West Green Street, Urbana, IL 61801-3080.

[1] For a review, see, e.g., Bose-Einstein Condensation in Atomic Gases, edited by M. Inguscio, S. Stringari, and C. E. Wieman (IOS Press, Amsterdam, 1999).

[2] D. A. W. Hutchinson, E. Zaremba, and A. Griffin, Phys. Rev. Lett. 78, 1842 (1997).

[3] V. V. Kocharovsky, Vl. V. Kocharovsky, and M. O. Scully, Phys. Rev. Lett. 84, 2306 (2000), and references therein.

[4] D. S. Jin, M. R. Matthews, J. R. Ensher, C. E. Wieman, and E. Cornell, Phys. Rev. Lett. 78, 764 (1997).

[5] D. N. Stamper-Kurn and H.-J. Miesner, S. Inouye, M. R. Andrews, and W. Ketterle, Phys. Rev. Lett. 81, 500 (1998).

[6] M. R. Matthews, B. P. Anderson, P. C. Haljan, D. S. Hall, C.E. Wieman, and E. A. Cornell, Phys. Rev. Lett. 83, 2498 (1999); K. W. Madison, F. Chevy, W. Wohlleben, and J. Dalibard, Phys. Rev. Lett. 84, 806 (2000); J. R. AboShaeer, C. Raman, J. M. Vogels, and W. Ketterle, Science 292, 476 (2001).
[7] P. O. Fedichev and G. V. Shlyapnikov, Phys. Rev. A 60, R1779 (1999).

[8] D. Pines and Ph. Nozières, The Theory of Quantum Liquids (Benjamin, New York, 1996).

[9] Y. Castin and R. Dum, Phys. Rev. Lett. 79, 3553 (1997).

[10] S. A. Morgan, J. Phys. B 33, 3847 (2000); M. Olshanii and L. Pricoupenko, cond-mat/0101275.

[11] Yu. Kagan and B. Svistunov, Phys. Rev. Lett. 79, 3331 (1997).

[12] M. J. Davis, S. A. Morgan, and K. Burnett, Phys. Rev. Lett. 87, 160402 (2001).

[13] M. J. Steel, M. K. Olsen, L. I. Plimak, P. D. Drummond, S. M. Tan, M. J. Collett, D. F. Walls, and R. Graham, Phys. Rev. A 58, 4824 (1998).

[14] M. Girardeau and R. Arnowitt, Phys. Rev. 113, 755 (1959).

[15] C. Gardiner, Phys. Rev. A 56, 1414 (1997).

[16] Y. Castin and R. Dum, Phys. Rev. A 57, 3008 (1998).

[17] We neglect here border effects appearing when the condensate mode is empty.

[18] A. Sinatra, Y. Castin, and C. Lobo, J. Mod. Opt. 47, 2629-2644 (2000).

[19] Note that this should not be the case for the exact Wigner distribution: e.g., $\left\langle N_{0}\right\rangle$ should be equal to $\left\langle\hat{N}_{0}\right\rangle+1 / 2$; the variances should differ by $1 / 4$.

[20] A. Sinatra, C. Lobo, and Y. Castin (to be published).

[21] This algorithm generates the probability distribution of the number of condensed particles $P\left(N_{0}\right)$. We checked in 1D and $2 \mathrm{D}$ that the first two moments of the obtained distributions reproduce the results of the Bogoliubov theory, which validates the approximation of $P\left(N_{0} \mid \psi_{\perp}\right)$ by a $\delta$. For the ideal Bose gas we successfully tested our result for $P\left(N_{0}\right)$ against the exact one; see [20].

[22] This damping does not occur for a single realization: our 1D model exhibits a collapse more than a damping.

[23] A necessary condition for the cutoff imposed by the discretization not to affect the predictions of the truncated Wigner method is that (i) the spatial step of the grid is smaller than both the healing length $\xi$ and the thermal wavelength $\lambda$ and (ii) the mean field due to the added noise in the Wigner representation remains small as compared to the real mean field; that is, the number of "empty" modes in the simulation is smaller than the number of particles. In 3D all these conditions are compatible for a dilute $\left(\rho|a|^{3} \ll 1\right)$ and degenerate $\left(\rho \lambda^{3} \gg 1\right)$ Bose gas, where $a$ is the $s$-wave scattering length and $\rho$ is the spatial density. A more detailed discussion is given in [20]. 